\title{
First-phase insulin secretion is positively correlated with alanine aminotransferase in young adults
}

\author{
Chung-Ze Wu ${ }^{1,2, A, C, D, F}$, Chang-Hsun Hsieh ${ }^{3, B, C, F}$, Chieh-Hua Lu ${ }^{3, B, C, F}$, Dee Pei ${ }^{4,5, A, C, E}$, Jin-Shuen Chen ${ }^{6,7, A-C, E, F}$, Yen-Lin Chen ${ }^{8, A-C, E, F}$ \\ ${ }^{1}$ Division of Endocrinology and Metabolism, Department of Internal Medicine, School of Medicine, College of Medicine, Taipei Medical University, Taiwan \\ ${ }^{2}$ Division of Endocrinology and Metabolism, Department of Internal Medicine, Shuang Ho Hospital, Taipei Medical University, New Taipei City, Taiwan \\ ${ }^{3}$ Division of Endocrinology and Metabolism, Department of Internal Medicine, Tri-Service General Hospital, National Defense Medical Center, Taipei, Taiwan \\ ${ }^{4}$ School of Medicine, College of Medicine, Fu Jen Catholic University, New Taipei City, Taiwan \\ ${ }^{5}$ Division of Endocrinology and Metabolism, Department of Internal Medicine, Fu Jen Catholic University Hospital, New Taipei City, Taiwan \\ ${ }^{6}$ Department of Medical Education and Research, Kaohsiung Veterans General Hospital, Taiwan \\ ${ }^{7}$ Division of Nephrology, Department of Internal Medicine, Tri-Service General Hospital, National Defense Medical Center, Taipei, Taiwan \\ ${ }^{8}$ Department of Pathology, Cardinal Tien Hospital, Fu Jen Catholic University, School of Medicine, New Taipei City, Taiwan \\ A - research concept and design; B - collection and/or assembly of data; $C$ - data analysis and interpretation; \\ $\mathrm{D}$ - writing the article; $\mathrm{E}$ - critical revision of the article; $\mathrm{F}$ - final approval of the article
}

\section{Address for correspondence}

Jin-Shuen Chen

E-mail: dgschen@vghks.gov.tw

\section{Funding sources}

The study was supported by the Cardinal Tien Hospital (grant No. CTH105A-2D06).

\section{Conflict of interest}

None declared

\section{Acknowledgements}

This manuscript was edited by Wallace Academic Editing. Authors thank all study participants in MJ Health Screening Centers, Taiwan.

Received on April 18, 2020

Reviewed on September 23, 2020

Accepted on 0ctober 8, 2020

Cite as

Wu CZ, Hsieh CH, Lu CH, Pei D, Chen JS, Chen YL.

First-phase insulin secretion is positively correlated with alanine aminotransferase in young adults. Adv Clin Exp Med. 2021;30(1):35-40. doi:10.17219/acem/128229

DOI

10.17219/acem/128229

\section{Copyright}

Copyright by Author(s)

This is an article distributed under the terms of the

Creative Commons Attribution 3.0 Unported (CC BY 3.0)

(https://creativecommons.org/licenses/by/3.0/)

\section{Abstract}

Background. Type 2 diabetes (T2D) is known to be one of the most prevalent diseases, and its prevalence is significantly associated with age and metabolic syndrome (MetS). Few studies have been conducted on liver function, MetS and insulin secretion among young adults.

Objectives. In the present study, we explored the relationship between the liver function enzyme - alanine aminotransferase (ALT) - and first-phase insulin secretion (FPIS) among young adults.

Material and methods. There were 22,971 men and 28,740 women, aged 18-27 years, assigned to subgroups according to the presence of MetS and quartiles of ALT values. Simple correlation was applied to evaluate their relationship. The difference between the slopes of these relationships and FPIS were statistically analyzed with Chris's calculator.

Results. Most values for metabolic parameters, including ALT and FPIS, were determined to be relatively high in individuals with MetS. By contrast, individuals with MetS had lower high-density-lipoprotein cholesterol (HDL-C) counts and FPIS. Similar results were observed in the quartiles of ALT. Significant positive results were also found in the linear model. Depending on the ALT level, the slope change of FPIS still demonstrated a positive correlation between ALT and FPIS. This correlation was stronger for men than for women.

Conclusions. A positive correlation between ALT and FPIS exists among young adults. Moreover, this correlation was stronger for men than for women. Both the cause and the effect require further investigation.

Key words: metabolic syndrome, alanine aminotransferase, first-phase insulin secretion 


\section{Introduction}

With the increasing prevalence of obesity, metabolic syndrome (MetS) is also coming to prominence as a serious health problem among adolescents and children worldwide. ${ }^{1}$ Following obesity, the prevalence of type 2 diabetes (T2D) has been increasing drastically in Taiwan as well as in many other countries. Moreover, among the factors contributing to the leading causes of mortality in Taiwan, T2D has remained among the top 5 for many years. ${ }^{2,3}$ Accordingly, patients, physicians, and health agencies worldwide have identified childhood obesity as a critical health concern. Children with obesity exhibit a higher risk for developing other diseases - some examples of which are coronary artery disease, fatty liver, polycystic ovary syndrome, and hypertension - not only during childhood, but throughout their lives. ${ }^{4}$ It is well-known that fatty liver is closely and bi-directionally related to MetS and T2D. Currently, the general consensus is that fatty liver is the hepatic manifestation of MetS. ${ }^{5}$

Abnormal liver function is often seen in clinical practice. Among the leading causes of abnormal alanine aminotransferase (ALT), nonalcoholic fatty liver disease is the most common, exhibiting a prevalence of $50-90 \%{ }^{6}$ Viral hepatitis is another disease that is endemic in Taiwan. Hepatitis B viral infection has been reported in the literature to have an estimated prevalence of $15-20 \%{ }^{7,8}$ Patients with hepatitis B also have abnormal liver function. Thus, it is necessary to clarify the relationships between abnormal liver function and T2D.

Three major pathophysiological mechanisms are generally considered to be potential causes of glucose intolerance: reduction of insulin activity (or insulin resistance - IR), insulin secretion and glucose effectiveness. ${ }^{9}$ In addition, there are 2 phases of insulin secretion, namely, first-phase insulin secretion (FPIS) and second-phase insulin secretion. ${ }^{10,11}$ Notably, an impairment of insulin secretion is related to a deterioration of liver function. ${ }^{12}$ Consequently, clarifying the underlying causes behind T2D and FPIS is crucial for health providers and future treatment strategy. So far, few studies have evaluated the relationship between ALT and FPIS in young adults. The present study was conducted with the underlying aim of exploring the correlation between FPIS and ALT among young adults.

\section{Methods}

\section{Study participants}

This study employed random sampling to enroll 22,971 men and 28,740 women aged 18-27 years from private clinics and local hospitals in Taiwan. The MJ Health Screening Centers, which are a chain of private clinics in Taiwan, offer their members regularly scheduled health examinations. They provided data solely for the purpose of research, and approval was obtained for the protocol of this study from the MJ Health Screening Center Institutional Review Board. All participants in this study remained anonymous and provided informed consent. The definition of obesity in this study was a body mass index $(B M I) \geq 25 \mathrm{~kg} / \mathrm{m}^{2}$. Participants with obesity were excluded if they took any medications that have been demonstrated to affect blood pressure or glucose and lipid levels. The patients were categorized into 2 groups - without MetS (MetS(-) group) and with MetS (MetS(+) group). The presence of MetS was defined according to World Health Organization (WHO) criteria. ${ }^{13}$ Finally, 768 men and 794 women were included in the MetS(+) group. Furthermore, all participants were divided into quartiles based on their ALT levels for advanced analysis.

On the day of the study, a senior member of the nursing staff recorded the medical history of all participants, including relevant information regarding any medications currently being taken, and conducted a physical examination. Horizontal measurements were performed at the location of the natural waist to record waist circumference (WC). To calculate BMI, the body weight (in kilograms) of participants was divided by the square of their height (in meters). Standard mercury sphygmomanometers were employed to perform measurements of diastolic blood pressure (DBP) and systolic blood pressure (SBP) measured on the right arm of the participants while they were seated.

\section{Laboratory biochemistry measurement}

Blood samples were collected for biochemical analysis after $10 \mathrm{~h}$ of fasting. Within $1 \mathrm{~h}$ of blood collection, the plasma was extracted, after which it was kept at $30^{\circ} \mathrm{C}$ until the lipid profile and fasting plasma glucose (FPG) assays were performed. A glucose oxidase approach (YSI 203 glucose analyzer; Yellow Springs Instruments, Yellow Springs, USA) was employed for measuring FPG. Measurements of triglyceride (TG) levels and total cholesterol were conducted using a Fuji Dri-Chem 3000 analyzer (Fuji Photo Film, Tokyo, Japan) and a dry, multilayer analytical slide method. Analyses of serum low-density lipoprotein cholesterol (LDL-C) and high-density lipoprotein cholesterol (HDL-C) concentrations were performed after dextran sulfate precipitation through an enzymatic cholesterol assay.

\section{Assessment of FPIS}

We used the equation derived from our other groups, ${ }^{14}$ which are listed below (international units). To demonstrate the reliability of our equations, a short statement is given here. When performing this study, data of $70 \%$ of the participants was used to build the equations and 
data the remaining $30 \%$ was used for external validation. The accuracy of the equations could therefore be tested.

In total, there were 186 subjects enrolled. The FPIS was measured using a frequently sampled intravenous glucose tolerance test. The R-value between the measured and calculated FPIS was 0.671 ( $\mathrm{p}<0.001) .{ }^{14}$ The equation is shown below:

$$
\mathrm{FPIS}=10^{(1.477-0.119 \times \mathrm{FPG}+0.079 \times \mathrm{BMI}-0.523 \times \mathrm{HDL}-\mathrm{C})}
$$

\section{Statistical analyses}

The IBM SPSS v. 19.0 software (IBM Inc., Armonk, USA) was used in the study to conduct all of the statistical analyses. The resulting data is provided as means \pm standard deviation (SD). Levene's test and the Kolmogorov-Smirnov test were applied to all data to assess the homogeneity of variance and normal distribution, respectively. If an abnormal distribution of data was found, then the data was subjected to log transformation before analysis. To identify differences between groups with and without MetS, a t-test was conducted. The study also used one-way analysis of variance (ANOVA) to assess the difference between the mean values of the 4 groups. Bonferroni post hoc analysis was performed for intergroup comparisons. A simple correlation was adopted in order to assess the correlation between 2 independent variables. Concurrently, the slopes of these relationships could also be obtained. We adopted $0 \%$ and $100 \%$ as the lowest and highest FPIS values, respectively, with values between these 2 extremes being calculated as the corresponding percentage. To compare the slopes between these 2 lines in order to determine whether they differed significantly, we utilized Chris's calculator. ${ }^{15}$

\section{Results}

Table 1 presents the demographics of our study cohort. Regardless of gender, the participants in the MetS(+) group exhibited unfavorable results for MetS-related factors, including BMI, WC, SBP, DBP, TG, FPG, HDL-C, and LDLC. In addition, the participants in the MetS(+) group were determined to have higher levels of both FPIS and ALT, which constituted the most critical factors. As already mentioned, all participants were subdivided on the basis of the quartiles of ALT results into 4 groups. Notably, for both sexes, participants with higher ALT levels had lower HDL-C levels but higher SBP, WC, FPG, DBP, BMI, TG, and LDL-C levels (Table 2). A scatter plot of the correlation of ALT and log transformation of FPIS is presented in Fig. 1. The correlation coefficient $(\mathrm{r})$ values were 0.349 for men and 0.133 for women. The correlations for both genders were statistically significant $(\mathrm{p}<0.001)$. Figure 2 presents the different slopes of log transformation of FPIS in men and women. As described in the Methods section, FPIS was transformed into a percentage of the maximum value (100\%). Through a comparison of the genders regarding changes to the FPIS slope according to ALT level, we discovered that men had a higher slope than women.

\section{Discussion}

In the current study, our data demonstrated that there is a positive relationship between ALT and FPIS in Chinese young adults. This is the first study, to our knowledge, to present these results for a group of relative healthy, nonobese subjects without any possible confounding effects

Table 1. General characteristics of subjects without and with metabolic syndrome (MetS) according to gender

\begin{tabular}{|c|c|c|c|c|c|c|}
\hline \multirow{3}{*}{ Parameter } & \multicolumn{6}{|c|}{ Group } \\
\hline & \multicolumn{3}{|c|}{ Men } & \multicolumn{3}{|c|}{ Women } \\
\hline & MetS(-) & MetS(+) & p-value & MetS(-) & MetS(+) & $\mathrm{p}$-value \\
\hline $\mathrm{N}$ & 21,236 & 1735 & & 28,225 & 515 & \\
\hline Age [years] & $24.21 \pm 2.55$ & $24.58 \pm 2.54$ & $<0.001$ & $24.23 \pm 2.42$ & $24.17 \pm 2.61$ & 0.596 \\
\hline $\mathrm{BMI}\left[\mathrm{kg} / \mathrm{m}^{2}\right]$ & $22.4 \pm 3.3$ & $28.7 \pm 4.4$ & $<0.001$ & $20.1 \pm 2.8$ & $28.9 \pm 5.3$ & $<0.001$ \\
\hline $\mathrm{WC}[\mathrm{cm}]$ & $76.3 \pm 8.1$ & $92.0 \pm 10.4$ & $<0.001$ & $65.8 \pm 6.2$ & $84.5 \pm 10.9$ & $<0.001$ \\
\hline $\mathrm{SBP}[\mathrm{mm} \mathrm{Hg}]$ & $118.3 \pm 12.4$ & $133.3 \pm 12.8$ & $<0.001$ & $106.6 \pm 11.1$ & $125.6 \pm 14.1$ & $<0.001$ \\
\hline $\mathrm{DBP}[\mathrm{mm} \mathrm{Hg}]$ & $68.2 \pm 8.8$ & $77.1 \pm 10.3$ & $<0.001$ & $62.8 \pm 8.1$ & $73.4 \pm 10.7$ & $<0.001$ \\
\hline $\mathrm{FPG}[\mathrm{mg} / \mathrm{dL}]$ & $93.51 \pm 7.38$ & $101.57 \pm 17.98$ & $<0.001$ & $89.82 \pm 7.25$ & $101.90 \pm 23.31$ & $<0.001$ \\
\hline $\mathrm{TG}[\mathrm{mg} / \mathrm{dL}]$ & $86.30 \pm 42.12$ & $172.61 \pm 72.12$ & $<0.001$ & $67.92 \pm 29.26$ & $146.50 \pm 66.58$ & $<0.001$ \\
\hline $\mathrm{HDL}-\mathrm{C}[\mathrm{mg} / \mathrm{dL}]$ & $51.81 \pm 11.54$ & $39.58 \pm 8.20$ & $<0.001$ & $62.75 \pm 14.10$ & $43.32 \pm 7.74$ & $<0.001$ \\
\hline LDL-C [mg/dL] & $105.02 \pm 28.37$ & $117.97 \pm 32.58$ & $<0.001$ & $97.80 \pm 26.50$ & $112.08 \pm 30.39$ & $<0.001$ \\
\hline $\mathrm{ALT}[\mathrm{U} / \mathrm{L}]$ & $27.8 \pm 25.8$ & $57.6 \pm 46.0$ & $<0.001$ & $16.0 \pm 14.7$ & $34.3 \pm 32.6$ & $<0.001$ \\
\hline Log_FPIS [ $\mu \mathrm{U} / \mathrm{min}]$ & $1.928 \pm 0.327$ & $2.534 \pm 0.374$ & $<0.001$ & $1.624 \pm 0.322$ & $2.496 \pm 0.452$ & $<0.001$ \\
\hline
\end{tabular}

Data presented as means \pm standard deviation (SD); BMI - body mass index; WC - waist circumference; SBP - systolic blood pressure; DBP - diastolic blood pressure; FPG - fasting plasma glucose; TG - triglyceride; HDL-C - high-density-lipoprotein cholesterol; LDL-C - low-density-lipoprotein cholesterol; ALT - alanine aminotransferase; Log FPIS - log transformation of first-phase insulin secretion. 
Table 2. Categorization of alanine aminotransferase levels from low to high

\begin{tabular}{|c|c|c|c|c|c|c|c|c|c|}
\hline Parameter & \multicolumn{2}{|l|}{ ALT1 } & \multicolumn{2}{|l|}{ ALT2 } & \multicolumn{2}{|l|}{ ALT3 } & \multicolumn{2}{|l|}{ ALT4 } & $p$-value \\
\hline \multicolumn{10}{|c|}{ Men } \\
\hline $\mathrm{n}$ & 5,414 & & 6,230 & & 5,759 & & 5,531 & & \\
\hline $\mathrm{ALT}[\mathrm{U} / \mathrm{L}]$ & $11.6 \pm 2.0$ & $2,3,4$ & $17.8 \pm 2.0$ & $1,3,4$ & $27.0 \pm 3.7$ & $1,2,4$ & $65.0 \pm 41.4$ & $1,2,3$ & $<0.001$ \\
\hline Age [years] & $24.63 \pm 2.38$ & $2,3,4$ & $24.55 \pm 2.45$ & $1,3,4$ & $24.15 \pm 2.57$ & 1,2 & $25.63 \pm 2.69$ & 1,2 & $<0.001$ \\
\hline BMI $\left[\mathrm{kg} / \mathrm{m}^{2}\right]$ & $20.8 \pm 2.4$ & $2,3,4$ & $21.9 \pm 2.8$ & $1,3,4$ & $23.2 \pm 3.3$ & $1,2,4$ & $25.6 \pm 4.4$ & $1,2,3$ & $<0.001$ \\
\hline$W C[\mathrm{~cm}]$ & $72.22 \pm 6.36$ & $2,3,4$ & $75.20 \pm 7.25$ & $1,3,4$ & $78.42 \pm 9.30$ & $1,2,4$ & $84.08 \pm 10.59$ & $1,2,3$ & $<0.001$ \\
\hline $\mathrm{SBP}[\mathrm{mm} \mathrm{Hg}]$ & $117 \pm 12$ & $2,3,4$ & $118 \pm 13$ & $1,3,4$ & $120 \pm 13$ & $1,2,4$ & $120 \pm 13$ & $1,2,3$ & $<0.001$ \\
\hline $\mathrm{DBP}[\mathrm{mm} \mathrm{Hg}]$ & $67 \pm 9$ & $2,3,4$ & $68 \pm 9$ & $1,3,4$ & $69 \pm 9$ & $1,2,4$ & $71 \pm 10$ & $1,2,3$ & $<0.001$ \\
\hline $\mathrm{FPG}[\mathrm{mg} / \mathrm{dL}]$ & $93 \pm 7$ & $2,3,4$ & $94 \pm 10$ & 1,4 & $94 \pm 8$ & 1,4 & $95 \pm 9$ & $1,2,3$ & $<0.001$ \\
\hline $\mathrm{TG}[\mathrm{mg} / \mathrm{dL}]$ & $74 \pm 31$ & $2,3,4$ & $81 \pm 39$ & $1,3,4$ & $96 \pm 48$ & $1,2,4$ & $121 \pm 65$ & none & $<0.001$ \\
\hline $\mathrm{HDL}-\mathrm{C}[\mathrm{mg} / \mathrm{dL}]$ & $52.3 \pm 11.3$ & 3,4 & $52.2 \pm 11.5$ & 3,4 & $50.8 \pm 12.1$ & $1,2,4$ & $48.1 \pm 11.6$ & $1,2,3$ & $<0.001$ \\
\hline $\mathrm{LDL}-\mathrm{C}[\mathrm{mg} / \mathrm{dL}]$ & $96.4 \pm 25.7$ & $2,3,4$ & $101.9 \pm 27.0$ & $1,3,4$ & $108.9 \pm 28.1$ & $1,2,4$ & $117.1 \pm 30.6$ & $1,2,3$ & $<0.001$ \\
\hline Log_FPIS $[\mu \mathrm{U} / \mathrm{min}]$ & $1.796 \pm 0.265$ & $2,3,4$ & $1.885 \pm 0.300$ & $1,3,4$ & $2.006 \pm 0.345$ & $1,2,4$ & $2.215 \pm 0.409$ & $1,2,3$ & $<0.001$ \\
\hline \multicolumn{10}{|c|}{ Women } \\
\hline$n$ & 7,762 & & 8,070 & & 6,046 & & 6,853 & & \\
\hline $\mathrm{ALT}[\mathrm{U} / \mathrm{L}]$ & $8.7 \pm 1.4$ & $2,3,4$ & $11.9 \pm 0.8$ & $1,3,4$ & $15.2 \pm 1.1$ & $1,2,4$ & $31.2 \pm 26.0$ & $1,2,3$ & $<0.001$ \\
\hline Age [years] & $24.34 \pm 2.37$ & $2,3,4$ & $24.34 \pm 2.38$ & 1 & $24.22 \pm 2.43$ & 1 & $23.99 \pm 2.52$ & 1 & $<0.001$ \\
\hline BMI $\left[\mathrm{kg} / \mathrm{m}^{2}\right]$ & $19.7 \pm 2.3$ & $2,3,4$ & $19.9 \pm 2.5$ & $1,3,4$ & $20.3 \pm 2.9$ & $1,2,4$ & $21.4 \pm 4.1$ & $1,2,3$ & $<0.001$ \\
\hline$W V[\mathrm{~cm}]$ & $65.0 \pm 5.3$ & $2,3,4$ & $65.5 \pm 5.7$ & $1,3,4$ & $66.2 \pm 6.4$ & $1,2,4$ & $68.4 \pm 8.8$ & $1,2,3$ & $<0.001$ \\
\hline $\mathrm{SBP}[\mathrm{mm} \mathrm{Hg}]$ & $106 \pm 11$ & 3,4 & $107 \pm 11$ & 4 & $107 \pm 11$ & 1,4 & $108 \pm 12$ & $1,2,3$ & $<0.001$ \\
\hline $\mathrm{DBP}[\mathrm{mm} \mathrm{Hg}]$ & $63 \pm 8$ & 4 & $63 \pm 8$ & 4 & $63 \pm 8$ & none & $64 \pm 9$ & 1,2 & $<0.001$ \\
\hline $\mathrm{FPG}[\mathrm{mg} / \mathrm{dL}]$ & $90 \pm 7$ & 4 & $90 \pm 7$ & 4 & $90 \pm 7$ & 4 & $91 \pm 11$ & $1,2,3$ & $<0.001$ \\
\hline $\mathrm{TG}[\mathrm{mg} / \mathrm{dL}]$ & $65 \pm 26$ & 3,4 & $66 \pm 28$ & 3,4 & $69 \pm 32$ & $1,2,4$ & $78 \pm 40$ & $1,2,3$ & $<0.001$ \\
\hline $\mathrm{HDL}-\mathrm{C}[\mathrm{mg} / \mathrm{dL}]$ & $61 \pm 13$ & 2,3 & $63 \pm 14$ & 1,4 & $64 \pm 15$ & 1,4 & $62 \pm 15$ & 2,3 & $<0.001$ \\
\hline $\mathrm{LDL}-\mathrm{C}[\mathrm{mg} / \mathrm{dL}]$ & $95 \pm 25$ & $2,3,4$ & $97 \pm 27$ & 1,4 & $98 \pm 26$ & 1,4 & $102 \pm 28$ & $1,2,3$ & $<0.001$ \\
\hline Log_FPIS [ $\mu \mathrm{U} / \mathrm{min}]$ & $1.607 \pm 0.276$ & 4 & $1.604 \pm 0.302$ & 4 & $1.625 \pm 0.343$ & 4 & $1.732 \pm 0.435$ & $1,2,3$ & $<0.001$ \\
\hline
\end{tabular}

Data is presented as means \pm standard deviation (SD); ${ }^{1} \mathrm{p}<0.05$ vs ALT1; ${ }^{2} \mathrm{p}<0.05$ vs ALT2 $;{ }^{3} \mathrm{p}<0.05$ vs ALT3; ${ }^{4} \mathrm{p}<0.05$ vs ALT4; BMI - body mass index; WC - waist circumference; SBP - systolic blood pressure; DBP - diastolic blood pressure; FPG - fasting plasma glucose; TG - triglyceride; HDL-C - high-densitylipoprotein cholesterol; LDL-C - low-density-lipoprotein cholesterol; ALT - alanine aminotransferase; Log FPIS - log transformation of first-phase insulin secretion.
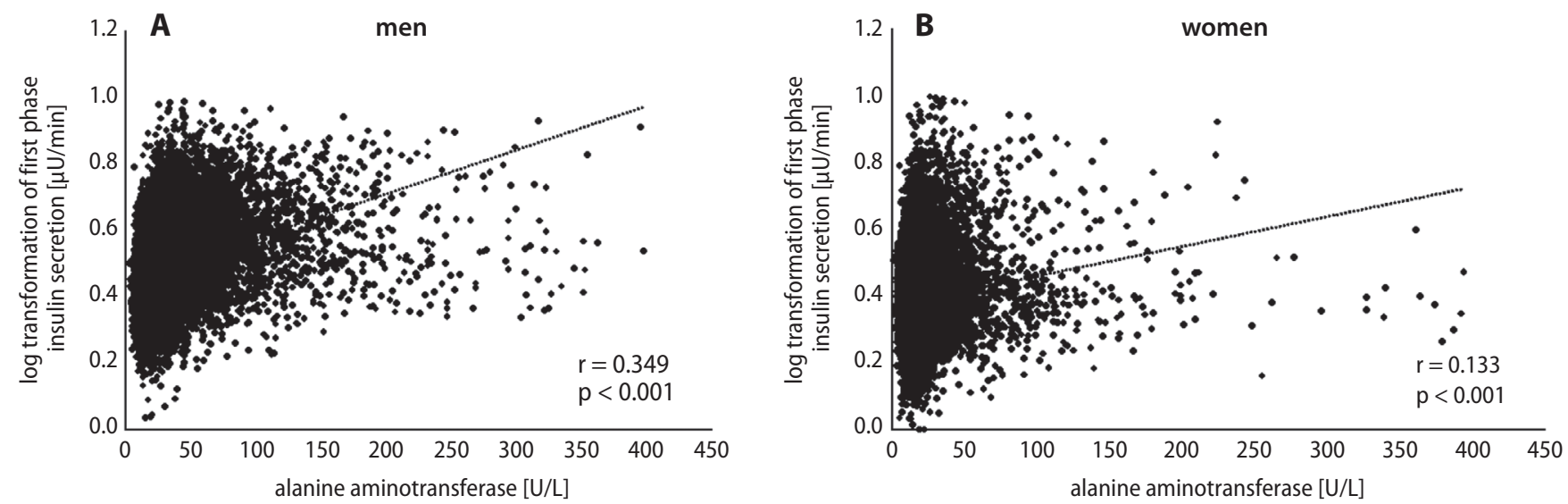

Fig. 1. Scatter plot of log-transformed first-phase insulin secretion and alanine aminotransferase levels in (A) men and (B) women

from drugs used for treating hypertension, dyslipidemia or T2D.

Few studies have evaluated the correlation between ALT and FPIS. Therefore, the relative issue is still debated.
By using the homeostasis model assessment, Hsiao et al. reported that both insulin secretion and sensitivity exhibited simultaneously significant impairment with increased ALT levels in 284 Chinese adults. ${ }^{12}$ Their findings contrast 


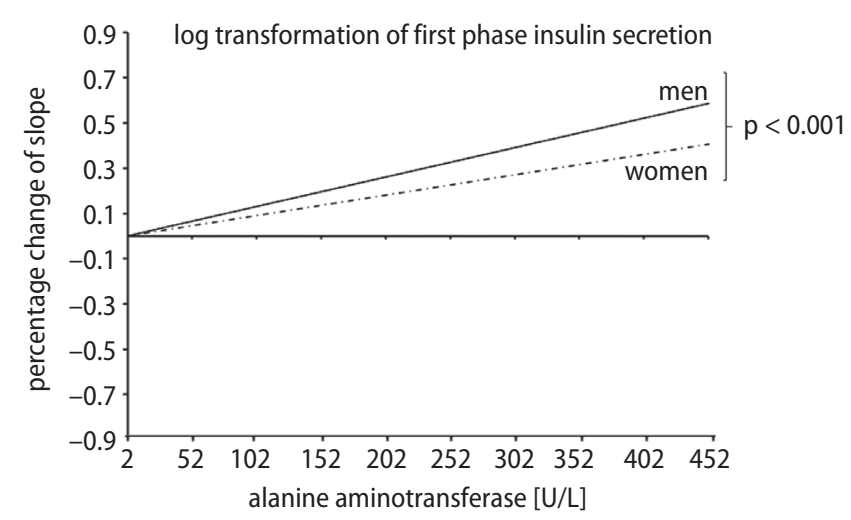

Fig. 2. Comparison of the genders regarding slope changes of first-phase insulin according to ALT levels

with ours. However, their study population was smaller than ours and they evaluated only participants who had FPG levels ranging from $100 \mathrm{mg} / \mathrm{dL}$ to $109 \mathrm{mg} / \mathrm{dL}$. In order to explain the discrepancy between these 2 different results, we hypothesize that obesity may be the link between ALT and FPIS. We can observe that individuals with obesity have higher ALT levels as well as elevated insulin secretion. Therefore, it could be postulated that people with higher ALT levels exhibit increased insulin secretion.

It is well-known that insulin secretion consists of 2 phases: FPIS and SPIS. ${ }^{10,11}$ After intravenous glucose infusion, glucose concentration increases rapidly. A biphasic response of insulin secretion is then observed, which comprises a sharp increase that reaches a peak. This stage is followed by a nadir between peaks and subsequently a slower increasing phase. Although the existence of FPIS is still under debate, a great deal of evidence supports it. For instance, Steiner et al. demonstrated that FPIS and SPIS play different roles in insulin secretion. ${ }^{16}$ Using a sophisticated method that employed a pancreatic clamp, they discovered that FPIS is crucial for suppressing the acute response of glucose stimulation. Even though this paper was published 30 years ago, it still constitutes a milestone in this topic. Furthermore, Kepner et al. suggested that the cascade involved in SPIS is different from that in FPIS. ${ }^{17}$ In SPIS, Cool-1/ßPIX operates as an exchange factor for guanine nucleotide to regulate insulin secretion, which is not the case in FPIS.

In our study, we discovered that participants with obesity had higher levels of insulin secretion. Kreisberg et al. might be the first group to report this observation. ${ }^{18}$ In 1968, Karam et al. further expounded on this relationship. ${ }^{19}$ They discovered that after glucose challenge, a more considerable acute insulin increase was noted in obese patients compared with healthy individuals. ${ }^{20}$ Because studies have indicated no impairment of insulin clearance, they concluded that this increase in insulin response must be due to higher levels of insulin secretion. Many studies conducted since then have also shown that individuals with obesity have superior cell function due to a greater cell mass compared with individuals without obesity. ${ }^{21-23}$
Recently, our group also validated this result by enrolling individuals with the same age and BMI. Our data suggested that BMI is positively related to FPIS in both men and women (R-value: 0.966 and 0.926 , respectively). It should be noted that this is the only reference, to our knowledge, that is directly related to the role of FPIS alone. ${ }^{24}$

The ALT levels are higher in obese individuals. This finding is the final component needed to clarify the link between BMI and FPIS. Abnormal liver function has an estimated prevalence of $10-21 \% .{ }^{25}$ Among the causes of abnormal ALT, the most common is nonalcoholic fatty liver disease, which accounts for $50-90 \%$ of cases. ${ }^{6}$ Fatty liver disease can cause either simple steatosis without inflammation or nonalcoholic steatohepatisis. ${ }^{26}$ The second most common cause, particularly in Taiwan, is viral hepatitis; this is because Taiwan is one of the most endemic areas for hepatitis. ${ }^{8}$ Research has determined that there is a strong association between obesity and nonalcoholic fatty liver disease, with approx. $80 \%$ of obese individuals reported as having have nonalcoholic fatty liver disease. ${ }^{27,28}$ Moreover, viral hepatitis $C$ was reported to have a correlation with obesity. ${ }^{29}$ Such research validates the finding that ALT levels are higher among individuals with obesity.

This study has some limitations worth noting. Firstly, because this is a cross-sectional study, recall and selection bias are inherent limitations. Further research may be required in order to overcome these limitations. Secondly, it may be disputed that our equation is not an accurate method for quantifying FPIS. However, this method has been validated and published with an R-value of 0.6-0.7. Therefore, we still believe that the present study elucidates the relationships between ALT and FPIS by enrolling a large study sample.

\section{Conclusions}

In conclusion, our study revealed that there is a positive relationship between FPIS and ALT in Chinese young adults.

\section{ORCID iDs}

Chung-Ze Wu (D) https://orcid.org/0000-0001-6118-6070 Chang-Hsun Hsieh (D) https://orcid.org/0000-0001-8722-3044 Chieh-Hua Lu (D) https://orcid.org/0000-0002-2179-9486 Dee Pei (D) https://orcid.org/0000-0003-3080-4248

Jin-Shuen Chen (D) https://orcid.org/0000-0002-8697-7968

Yen-Lin Chen (D) https://orcid.org/0000-0002-6381-4269

\section{References}

1. Poyrazoglu S, Bas F, Darendeliler F. Metabolic syndrome in young people. Curr Opin Endocrinol Diabetes Obes. 2014;21(1):56-63.

2. Ministry of Health and Welfare. ROC: Annual Statistics Report, 19942017. https://www.mohw.gov.tw/mp-2.html. Accessed April 4, 2018.

3. Tseng $\mathrm{CH}$, Chong CK, Heng LT, Tseng CP, Tai TY. The incidence of type 2 diabetes mellitus in Taiwan. Diabetes Res Clin Pract. 2000;50(Suppl 2): S61-S64. 
4. Barton M. Childhood obesity: A life-long health risk. Acta Pharmacol Sin. 2012;33(2):189-193.

5. Yki-Järvinen H. Non-alcoholic fatty liver disease as a cause and a consequence of metabolic syndrome. Lancet Diabetes Endocrinol. 2014; 2(11):901-910.

6. Sanyal AJ, American Gastroenterological Association. AGA technical review on nonalcoholic fatty liver disease. Gastroenterology. 2002; 123(5):1705-1725.

7. Chen CJ, Wang LY, Yu MW. Epidemiology of hepatitis B virus infection in the Asia-Pacific region. J Gastroenterol Hepatol. 2000;15(Suppl):E3-E6.

8. Chen DS, Kuo GC, Sung JL, et al. Hepatitis $C$ virus infection in an area hyperendemic for hepatitis B and chronic liver disease: The Taiwan experience. J Infect Dis. 1990;162(4):817-822.

9. Bergman M. Pathophysiology of prediabetes and treatment implications for the prevention of type 2 diabetes mellitus. Endocrine. 2013;43(3):504-513.

10. Caumo A, Luzi L. First-phase insulin secretion: Does it exist in real life? Considerations on shape and function. Am J Physiol Endocrinol Metab. 2004;287(3):E371-E385.

11. Polonsky KS, Sturis J, Bell GI. Seminars in Medicine of the Beth Israel Hospital, Boston. Non-insulin-dependent diabetes mellitus: A genetically programmed failure of the beta cell to compensate for insulin resistance. N Engl J Med. 1996;334(12):777-783.

12. Hsiao JY, Wang CL, Hsia PJ, et al. Decreased insulin secretion and insulin sensitivity are associated with liver function in subjects with fasting glucose between 100 and $109 \mathrm{mg} / \mathrm{dL}$ in Taiwanese population. Pancreas. 2007;35(4):343-347.

13. Alberti KG, Zimmet PZ. Definition, diagnosis and classification of diabetes mellitus and its complications. Part 1: Diagnosis and classification of diabetes mellitus provisional report of a WHO consultation. Diabet Med. 1998;15(7):539-553.

14. Lin JD, Hsu CH, Liang YJ, et al. The estimation of first-phase insulin secretion by using components of the metabolic syndrome in a Chinese population. Int J Endocrinol. 2015;2015:675245.

15. https://www.surrey.ac.uk/search?query=calculator\&op=Search.

16. Steiner KE, Mouton SM, Bowles CR, Williams PE, Cherrington AD. The relative importance of first- and second-phase insulin secretion in countering the action of glucagon on glucose turnover in the conscious dog. Diabetes. 1982;31(11):964-972.
17. Kepner EM, Yoder SM, Oh E, et al. Cool-1/ßPIX functions as a guanine nucleotide exchange factor in the cycling of $\mathrm{Cdc} 42$ to regulate insulin secretion. Am J Physiol Endocrinol Metab. 2011;301(6):E1072-E1080.

18. Kreisberg RA, Boshell BR, DiPlacido J, Roddam RF. Insulin secretion in obesity. N Engl J Med. 1967;276(6):314-319.

19. Karam JH, Grodsky GM, Forsham PH. Insulin secretion in obesity: Pseudodiabetes? Am J Clin Nutr. 1968;21(12):1445-1454.

20. Karam JH, Grodsky GM, Forsham PH. Excessive insulin response to glucose in obese subjects as measured by immunochemical assay. Diabetes. 1963;12:197-204.

21. Hanley AJ, Wagenknecht LE, D'Agostino RB Jr, Zinman B, Haffner SM. Identification of subjects with insulin resistance and beta-cell dysfunction using alternative definitions of the metabolic syndrome. Diabetes. 2003;52(11):2740-2747.

22. Klöppel G, Löhr M, Habich K, Oberholzer M, Heitz PU. Islet pathology and the pathogenesis of type 1 and type 2 diabetes mellitus revisited. Surv Synth Pathol Res. 1985;4(2):110-125.

23. van Haeften TW, Dubbeldam S, Zonderland ML, Erkelens DW. Insulin secretion in normal glucose-tolerant relatives of type 2 diabetic subjects: Assessments using hyperglycemic glucose clamps and oral glucose tolerance tests. Diabetes Care. 1998;21(2):278-282.

24. Lin JD, Hsu CH, Wu CZ, et al. Effect of body mass index on diabetogenesis factors at a fixed fasting plasma glucose level. PLoS One. 2018;13(1):e0189115.

25. Radcke S, Dillon JF, Murray AL. A systematic review of the prevalence of mildly abnormal liver function tests and associated health outcomes. Eur J Gastroenterol Hepatol. 2015;27(1):1-7.

26. Agrawal S, Dhiman RK, Limdi JK. Evaluation of abnormal liver function tests. Postgrad Med J. 2016;92(1086):223-234.

27. Marchesini G, Moscatiello S, Di Domizio S, Forlani G. Obesity-associated liver disease. J Clin Endocrinol Metab. 2008;93(11 Suppl 1):S74-S80.

28. Mota M, Banini BA, Cazanave SC, Sanyal AJ. Molecular mechanisms of lipotoxicity and glucotoxicity in nonalcoholic fatty liver disease. Metabolism. 2016;65(8):1049-1061.

29. Hu KQ, Kyulo NL, Esrailian E, et al. Overweight and obesity, hepatic steatosis, and progression of chronic hepatitis C: A retrospective study on a large cohort of patients in the United States. $J$ Hepatol. 2004;40(1):147-154. 